\begin{tabular}{|l|l|}
\hline $\begin{array}{l}\text { Postprint } \\
\text { Version }\end{array}$ & 1.0 \\
\hline Journal website & http://onlinelibrary.wiley.com/doi/10.1111/acer.12817/abstract \\
\hline Pubmed link & $\underline{\text { http://www.ncbi.nlm.nih.gov/pubmed/26247388 }}$ \\
\hline DOI & $10.1111 /$ acer.12817 \\
\hline
\end{tabular}

This is a NIVEL certified Post Print, more info at http://www.nivel.eu

\title{
Attentional Bias and Approach/Avoidance Tendencies Do Not Predict Relapse or Time to Relapse in Alcohol Dependency
}

\author{
Michelle Snelleman, Tim MichaËl Schoenmakers, Dike VAn De MHeEN
}

\begin{abstract}
Background

Implicit processes such as attentional bias (AB) and automatic approach/avoidance tendencies (AA) play a role in substance use disorders. Whether these processes can predict a relapse in alcohol-dependent patients is still unclear and must be examined in more detail than has been done previously. We aimed to establish whether $\mathrm{AB}$ and $\mathrm{AA}$ measured during treatment would predict relapse in alcohol-dependent patients. We also investigated whether these implicit processes predicted time to relapse better than a more common binary relapse variable.

Methods

A total of 50 alcohol-dependent outpatients undergoing treatment completed the study. Patients completed the Addiction Stroop Task, which assesses AB, and the relevant Stimulus-Response Compatibility Task, which measures AA. Time to relapse was assessed 1,2, and 3 months after the bias assessment.

Results

Twenty patients (40\%) relapsed during the follow-up period. The average time to relapse was 40 days after the first session. Overall, participants had an $\mathrm{AB}$ for alcohol-related stimuli and a tendency to avoid these stimuli. Neither relapse nor time to relapse was predicted by either bias type.

Conclusions

Although both $\mathrm{AB}$ and avoidance tendencies were present in our sample, these measures did not predict relapse or time to relapse in an outpatient alcoholdependent sample in the following 3 months. Future research should focus on studying the predictive value of these biases in the short term, for example, using ecological momentary assessment techniques to assess implicit processes shortly before a relapse.
\end{abstract}


Implicit processes have become a major field of interest in the study of substance use. So far, we know that both attentional bias (AB; i.e., selective attention for substance-related stimuli or cues) and approach/avoidance tendencies (AA; i.e., the tendency to move toward or away from substance-related cues) each play a role in substance use disorders (e.g., Mogg et al., 2005; Roefs et al., 2011). Several theoretical models have explained how these biases may play a causal role in the onset, maintenance, and/or recurrence of substance use disorders after a period of abstinence (Franken, 2003; Garland et al., 2011; Tiffany, 1990; Wiers et al., 2007). Because of $\mathrm{AB}$ toward substance-related cues, exposure to these cues eventually increases, which triggers learned responses to the cues. Some of these learned responses represent other implicit processes. One, for example, is related to the motor system: the tendency to move toward or away from substance-related cues, that is, AA (Garland et al., 2011). AB can be assessed using computerized tasks such as the Addiction Stroop (Cox et al., 2006), while AA are assessed with computer tasks measuring whether movements, symbolic or actual, toward substance-related stimuli are faster than toward neutral stimuli (e.g., Cousijn et al., 2011; Field et al., 2005; Schoenmakers et al., 2008).

Despite the current knowledge, the clinical relevance of $\mathrm{AB}$ and $\mathrm{AA}$ remains unclear. Both Field and colleagues (2014) and Christiansen and colleagues (2014) concluded in their narrative reviews that $\mathrm{AB}$ for substance-related cues is a reliable feature of substance use disorders. Those authors, however, did not recommend assessing $\mathrm{AB}$ in treatment settings, for example, to identify those at risk for relapse, because the available evidence is still ambiguous. Studies measuring AB for alcohol-related stimuli during treatment have either shown that $A B$ predicts relapse after discharge (Garland et al., 2012) or failed to find this relationship altogether (Field et al., 2013). There are currently no published review articles on the clinical relevance of AA because there have been few such studies conducted in the field. However, evidence exists that alcohol-related stimuli can trigger approach tendencies in heavy drinkers (e.g., Field et al., 2008; Ostafin and Palfai, 2006; Palfai and Ostafin, 2003; Wiers et al., 2009). Additionally, there seems to be a positive association between pretreatment drinking levels and approach tendencies in recently detoxified alcoholdependent patients (Barkby et al., 2012), but it remains unclear whether approach tendencies or avoidance tendencies are predictive of subsequent relapse.

To our knowledge, only 1 published study has assessed the predictive value of AA as measured during treatment on the occurrence of posttreatment relapse (Spruyt et al., 2013). In this study, the relevant Stimulus-Response Compatibility Task (RSRC Task; De Houwer et al., 2001) was used in an alcohol-dependent sample undergoing treatment. R-SRC bias scores were predictive of relapse 3 months later. Specifically, participants who showed a tendency to avoid, not approach, alcoholrelated pictures had an increased likelihood of relapsing (Spruyt et al., 2013). The authors proposed that alcohol-dependent patients who abstain may develop avoidance tendencies during early abstinence. Successful relapse prevention requires in-depth emotional processing of alcohol-related cues, making alcohol-related memory structures available for modification. This in-depth processing is not possible when alcohol-related cues are being avoided, keeping the maladaptive alcohol-related memories intact, and encountering alcohol-related cues will activate these maladaptive memories, increasing the risk of craving and relapse. This idea, however, contrasts with findings from avoidance-training studies in which training 
patients to avoid alcohol-related stimuli resulted in reduced relapse rates (Eberl et al., 2013, 2014; Wiers et al., 2011).

Until now, there is still no gold standard measure of relapse. There are various ways to define and to measure relapse. For example, relapse can be defined as returning to pretreatment drinking levels or as having a binge episode (i.e., having more than 4 of 5 standard drinks on a single occasion). Relapse is most often measured dichotomously, that is, a patient either relapsed or not. A number of possible measures have been proposed, for example, assessing the number of drinking days or the amount of alcohol consumed per drinking day, or a combination of these quantity and frequency measures (e.g., Marlatt and Witkiewitz, 2010). An alternative approach is to measure relapse in a continuous way, by assessing how many days a patient was abstinent prior to the relapse (Fuller, 1997). Christiansen and colleagues (2014) have recommended using time to relapse as outcome measure because it seems more appropriate from a clinical perspective. For instance, patients with a severe alcohol use disorder (AUD) often have a greater risk of relapse and of relapsing more quickly than patients with a mild or moderate AUD (e.g., Moos and Moos, 2006). Therefore, if we can use $A B$ and $A A$ to predict the amount of time before relapse, it should be a better indicator of treatment progress or success. A greater number of abstinent days would mean more treatment progress or even longterm treatment success. From a methodological perspective, it also makes sense to use a continuous outcome measure because this increases statistical power, as Christiansen and colleagues (2014) suggested (also see Fuller, 1997).

Therefore, the purpose of the current study was to investigate whether $\mathrm{AA}$ and/or $\mathrm{AB}$ can predict relapse in a sample of abstinent alcohol-dependent patients undergoing treatment. The second aim of this study was to investigate whether time to relapse is a better outcome measure for relapse than a binary yes/no variable.

We hypothesized that $\mathrm{AB}$ for alcohol-related cues would be present in abstinent alcohol-dependent patients and predict relapse. We also hypothesized that a greater $\mathrm{AB}$ would be predictive of a quicker relapse. Furthermore, we expected to find a relationship between AA and relapse. Specifically, based on the findings from Spruyt and colleagues (2013), we predicted that abstinent alcohol-dependent patients undergoing treatment would tend to avoid alcohol-related stimuli and that this avoidance tendency would predict relapse. Finally, we expected that greater avoidance of alcohol-related stimuli would be associated with a quicker relapse.

\section{MATERIALS AND METHODS}

\section{Participants}

Participants $(n=61)$ were recruited from 3 locations of an addiction treatment facility in the Netherlands. Two participants dropped out of the study before all data were collected. Our final sample consisted of 59 participants. Patients were eligible for the study after they had been abstinent from alcohol for at least 2 weeks. Recruitment took place in an outpatient detoxification program. This program consisted of 6 weeks of therapy, during which patients attended 2 group therapy sessions per week. The group sessions were led by 2 counselors who employed cognitive behavioral therapy and motivational interviewing techniques during these sessions. After this 6-week program, patients would continue with group therapy, terminate treatment, or start an individual treatment trajectory. 
The counselors of the treatment program determined whether a patient was eligible for the study based on our inclusion criteria. Inclusion criteria were the following: (i) a current diagnosis of alcohol dependence as defined in the DSM-IV (American Psychiatric Association 2000) and as determined by Section J of the Dutch version of the Composite International Diagnostic Interview (World Health Organization, 1997); (ii) an eighth-grade literacy level; and (iii) a period of abstinence of at least 2 weeks prior to the first appointment of the study. Patients were excluded if they (i) were diagnosed with an Axis II disorder according to the DSM-IV, (ii) were diagnosed with a disorder in the psychotic spectrum, or (iii) used other addictive substances (except nicotine) on a regular basis.

All participants provided informed consent before the start of the study. The Medical Ethical Committee declared that the Medical Research Involving Human Subjects Act (also known by its Dutch abbreviation WMO) does not apply to this research proposal. The Medical Ethical Committee had no objection to the execution of this research.

\section{Study Procedure}

The study presented here was part of a larger parent study, the procedure of which was as follows: Eligible patients were referred to the researcher by their counselor. The researcher then contacted the patient to explain the study in more detail and to provide an information leaflet about the study. The researcher contacted the patient again after 1 week to set up a first appointment.

The study consisted of 2 similar face-to-face sessions, scheduled 4 weeks apart during which computer tasks were administered that measured $\mathrm{AB}$ and $\mathrm{AA}$. A questionnaire on background variables (see Table 1) was administered in the first session, before the computer tasks.

\section{[TABLE 1]}

The first face-to-face session took place after the patient had been abstinent for at least 2 weeks. This 2 -week period was chosen to ensure that the participants did not experience any withdrawal symptoms during the sessions.

After completing the second session, participants received a financial compensation of $€ 25$. Our follow-up regarding the occurrence of relapse took place by telephone both 1 and 2 months after session 2 .

\section{Implicit Measures}

\section{Attentional Bias}

The Addiction Stroop Task (AST) was employed as a measure of AB (Cox et al., 2006) and was administered on an HP iPAQ Pocket PC (Hewlett Packard, Palo Alto, CA) running the Microsoft Windows Pocket PC operating system (Microsoft Corporation, Redmond, WA) in the same format as used by Waters and Li (2008). The iPAQ uses a pen-based touch screen system. The AST was programmed on the iPAQ to familiarize participants with using this device for a subsequent study (Snelleman M, Schoenmakers TM, van de Mheen D, in preparation) and has been administered successfully on an iPAQ (Waters and Li, 2008; Waters et al., 2012). In this task, words are presented on a screen in 3 different colors: red, green, and blue. Participants are instructed to ignore the meaning of the words and indicate in which color the word is shown by pressing the correct button on the screen. 
The AST consisted of 3 blocks of 33 trials each. The first block was the practice block and contained letter strings. The second and third blocks were the critical blocks. One block consisted of neutral words (Dutch equivalents of lamp, glue, computer, tape, pencil, scissors, desk, marker, paper, pen, eraser), while the other block consisted of alcohol-related words (Dutch equivalents of beer, wine, alcohol, cocktail, tequila, cognac, sherry, drink, liquor, gin, café). All words in the critical blocks were matched on word length and number of syllables. The presentation order of the 2 critical blocks was randomized at each session for each participant.

\section{Approach/Avoidance Tendencies}

The R-SRC Task was used to measure approach bias. The R-SRC has been shown to be a reliable predictor of hazardous drinking (Kersbergen et al., 2014). The R-SRC Task was programmed on a Dell laptop (Dell Inc., Round Rock, TX) with a 15.4" screen using Inquisit 3.0 (Millisecond 2012). In this task, participants are asked to either approach or avoid alcohol-related pictures by moving a manikin toward or away from these pictures.

The R-SRC Task consisted of 2 blocks: 1 approach alcohol block and 1 avoid alcohol block. Each block consisted of 10 practice trials and 56 critical trials. Stimuli consisted of 20 alcohol-related pictures and 20 neutral pictures (soda, water, tea, coffee). The context of the stimuli was either active (i.e., person holding the beverage) or passive (i.e., beverage on a white table). Pictures were presented in the center of the screen with a manikin $(12 \mathrm{~mm}$ high) positioned $33 \mathrm{~mm}$ above or below the picture. Participants were required to categorize the presented pictures by making an approach response (pressing the up or down key to move the manikin toward the picture) or an avoidance response (pressing the up or down key to move the manikin away from the picture). After making a correct response, the manikin would walk toward the picture (approach) or away from the picture (avoidance) for 1,000 ms. Following an incorrect response, a red cross appeared on the screen for $500 \mathrm{~ms}$, after which the next trial started. The order of blocks was counterbalanced among participants.

\section{Follow-Up Questionnaire on Relapse}

Follow-up information regarding relapse was gathered at the second face-to-face session and by telephone interviews 1 and 2, months after this second session. All participants were asked whether they had relapsed in the preceding month, how many drinking days they had had, and how much alcohol they had consumed during each drinking day. Relapse was defined as having at least 1 binge drinking episode. Binge drinking was defined as having 4 or more (for women) or 5 or more (for men) drinks on 1 single occasion (e.g., Corbin et al., 2014). Time to relapse was measured in number of abstinent days from the first face-to-face session until the day the participant relapsed.

\section{Statistical Analysis}

The D-measure scoring algorithm (Greenwald et al., 2003) was adapted to calculate the bias scores from the reaction times on the AST and the R-SRC. This algorithm is recommended when there are differences in baseline speed of responding because it protects against cognitive skill confounds. These confounds were likely to be present because our sample contained participants of varying ages and educational levels. 
First, response latencies faster than $300 \mathrm{~ms}$ or slower than 10 seconds were removed. Next, means and SDs of correct latencies per block and a pooled SD were calculated. Penalties were then applied for response errors. Response latencies on trials when participants made errors were replaced by the mean of the correct latencies for the relevant task block plus 2 standard deviations. After applying error penalties, mean response latencies was recalculated for all blocks (approach and avoidance block of the R-SRC Task; alcohol and neutral block of the AST).

R-SRC bias scores were calculated by subtracting the mean latency of the approach block from the mean latency of the avoidance block and dividing this difference score by the pooled SD. A positive score was therefore indicative of an approach bias, while a negative score indicated an avoidance bias. AB scores were calculated by subtracting the mean latency of the neutral block from the mean latency of the alcohol block and dividing this difference score by the pooled SD. A positive AB score thus indicated an AB.

\section{RESULTS}

\section{Demographics}

For the relapse analyses, we needed confirmation of a relapse having occurred at any time during the 3-month follow-up period, or confirmation of abstinence during the full 3 months. We could not obtain complete 3-month follow-up data for 9 participants $(15.3 \%)$. We have included only those participants with either complete follow-up data or, when follow-up was not complete, relapsed participants without complete follow-up in our analysis. Our final sample for analyses consisted of 50 participants.

Table 1 displays summary statistics for the whole sample $(n=50)$. There were no significant differences between abstainers and relapsers for age, gender ratio, education level, or number of previous treatments (all ps $>0.14$ ).

\section{Relapse}

In total, 20 participants relapsed during the 3-month follow-up period. Time to relapse was measured in days, with the mean time to relapse being 40 days $(\mathrm{SD}=22.19)$ after entering the study. Seven of those 20 participants had relapsed prior to session 2; therefore, all prospective analyses including data from session 2 were conducted without these early relapsers.

\section{Attentional Bias}

Table 2 describes the mean D-measure scores for the AST per group (abstainers vs. relapsers) for both sessions. Data from 2 participants (abstainers) at session 1 and data from 1 abstaining participant at session 2 were incomplete due to a technical malfunction of the personal digital assistant and were therefore not included in the analysis. Overall, AB was present in the whole sample at session $1, \mathrm{t}(47)=6.169, \mathrm{p}<0.001$. However, there were no differences in $\mathrm{AB}$ between relapsers and abstainers at session $1, \mathrm{t}(46)=0.591, \mathrm{p}=0.558$, Cohen's $\mathrm{d}=0.17$.

\section{[TABLE 2]}

At session 2, AB was still present in the whole sample, $\mathrm{t}(41)=2.52, \mathrm{p}=0.02$. However, there were no differences in $\mathrm{AB}$ between relapsers and abstainers at 
session $2, \mathrm{t}(40)=-0.62, \mathrm{p}=0.54$, Cohen's $\mathrm{d}=0.07$. Analyses using mean reaction time scores yielded similar results.

Spearman's rho correlation coefficient between $A B$ and time to relapse in the group of relapsers was also not significant $(\rho=-0.293, p=0.244)$. At session 2, correlation coefficients between AA and time to relapse again were not significant $(\rho=-0.301, p=0.20)$. Therefore, we did not perform any additional analyses using time to relapse as an outcome measure.

To check whether $\mathrm{AB}$ was stable over time, we calculated the association between $\mathrm{AB}$ scores at sessions 1 and 2. Pearson's correlation was low $(\mathrm{r}=0.29, \mathrm{p}=0.03)$, indicating that $\mathrm{AB}$ was not a stable trait in our sample. To check whether the stability of $\mathrm{AB}$ over time was influenced by patients who relapsed before session 2 , the analysis was repeated without those early relapsers. This resulted in a similarly low correlation $(\mathrm{r}=0.21)$.

\section{Approach/Avoidance Tendencies}

Table 2 presents the mean D-measure scores on the R-SRC by group (abstainers vs. relapsers) for both sessions. Overall, the results revealed a tendency to avoid alcoholrelated pictures, $\mathrm{t}(47)=-3.261, \mathrm{p}=0.002$. Again, no differences were found between relapsers and abstainers on the AA, $t(46)=0.248, p=0.805$, Cohen's $d=0.21$. At session 2, AA was no longer present in the whole sample, $\mathrm{t}(42)=-0.43, \mathrm{p}=0.67$. Additionally, there were no differences in AA between relapsers and abstainers at session $2, \mathrm{t}(41)=1.26, \mathrm{p}=0.21$, Cohen's $\mathrm{d}=0.41$. Analyses using mean reaction time scores yielded similar results.

Spearman's rho correlation coefficients between AA at session 1 and time to relapse were not significant $(\rho=-0.199, p=0.40)$. At session 2 , correlation coefficients between AA and time to relapse again were not significant $(\rho=0.205, p=0.39$ ). Therefore, no further analyses were performed with time to relapse.

We also checked whether AA was stable over time by calculating the association between AA scores at session 1 and AA scores at session 2. Pearson's correlation was low $(r=0.32, p=0.014)$, suggesting that AA was not a stable trait in our sample. To check whether the stability of AA over time was influenced by patients who relapsed before session 2 , the analysis was repeated without those early relapsers. This resulted in a similarly low correlation $(\mathrm{r}=0.31)$.

\section{DISCUSSION}

In this study, we investigated whether $\mathrm{AB}$ and $\mathrm{AA}$ could predict a relapse in abstinent alcohol-dependent outpatients. We found no evidence of the predictive value of these measures, even though our subjects did display both biases: an $\mathrm{AB}$ and avoidance tendencies. We also explored whether time to relapse would be a more sensitive outcome measure than a dichotomous relapse measure. However, AB and AA were also not predictive of time to relapse in our sample.

These findings are in line with conclusions drawn in the recent review by Christiansen and colleagues (2014). We could not find evidence that either AB or AA measured during treatment are predictors of relapse in the long term, in this case 3 months. However, we also did not find evidence for the suggestion made by Christiansen and colleagues (2014) that time to relapse may be a better outcome measure, as there was no relationship between time to relapse and $\mathrm{AA}$ or $\mathrm{AB}$ in our study. 
We have partially replicated the results found by Spruyt and colleagues (2013). Although, like them, we found an avoidance bias in a clinical sample of alcoholdependent patients using the R-SRC, we did not find evidence of the predictive value of this avoidance bias despite having identical follow-up periods. Additionally, there seems to be a discrepancy between alcoholic patients showing an avoidance bias (this study and Spruyt et al., 2013) and avoidance training having beneficial effects on relapse (Eberl et al., 2013; Wiers et al., 2011). We believe therefore that more research is needed in the underlying mechanisms of avoidance training. One major difference between our study and that of Spruyt and colleagues (2013) is that we recruited our subjects from an outpatient facility, whereas Spruyt and his colleagues recruited their sample from an inpatient detoxification facility. Furthermore, although we employed a broader definition of relapse, we obtained lower relapse rates. These differences indicate that the Spruyt and colleagues' study (2013) utilized a more severe sample and further suggest that the severity of dependence may differentially impact the relationship between AA and relapse. Finally, as we measured $\mathrm{AB}$ and not attentional control, we were not able to replicate the finding by Spruyt and colleagues (2013) that the relationship between R-SRC scores and relapse was reliable in those low in attentional control. Future research should investigate the joint contribution of AA and attentional control in relapse. Another suggestion for future research is to examine the joint contribution of AA and $\mathrm{AB}$ in predicting relapse.

There was no evidence to support the use of time to relapse as an outcome measure in our sample. This finding, however, should be interpreted with caution. We did find a moderate correlation between $\mathrm{AB}$ and time to relapse $(\rho=-0.293)$, but this correlation was not significant. The correlation between AA and time to relapse was also not significant $(\rho=-0.199)$, but this correlation is in the same direction as reported by Spruyt and colleagues (2013). This could be due to too little variation in the outcome measure, time to relapse, or to the small number of relapsers $(n=20)$. Therefore, future studies should further examine time to relapse as an outcome measure in larger samples to further test its relevance. Additionally, we defined relapse as having at least 1 binge episode. It is possible that a more stringent definition of relapse would show more distinctive results on $\mathrm{AA}$ and $\mathrm{AB}$ and their ability to predict relapse. Future research could incorporate multiple measures of relapse (e.g., number of binge episodes, relapse into pretreatment drinking levels, and number of relapses) to test this idea.

One limitation of the current study is the small sample size. Small samples obstruct finding significant small effects. However, our study's aim was to find clinical relevant effects, rather than merely theoretically interesting differences. Clinical relevance would have been demonstrated when effect sizes had been bigger, making it possible to predict relapse on an individual level, or at the very least on very small subsets of patients. As we did not find evidence for the predictive value of AA and $\mathrm{AB}$ in a small sample, we believe that measuring these biases during treatment has no clinical value in the assessment of potential relapse in individual patients. In addition, our study sample was rather homogeneous, because we included patients that had all been referred to the outpatient treatment program. When a study's aim is not clinical, but theoretical, it should be taken into account that small homogeneous samples may also lead to a rather low variance in bias and relapse scores. When one's 
aim is to detect theoretically relevant effects, a broader range of patients may lead to bigger effect sizes.

Another way forward in this type of research is varying the timing of measurement of $\mathrm{AA}$ and $\mathrm{AB}$. The first assessment of AA and $\mathrm{AB}$ occurred when participants had been abstinent for at least 2 weeks. It is possible that early abstinence or treatment alters these biases, obscuring their predictive value. In addition, measuring attentional and approach biases in the clinic and then predicting long-term relapse may not be the best strategy. Both biases can change over time, as we observed in our study. For example, the associations between bias scores from the 2 face-to-face sessions were weak. The strength of $\mathrm{AB}$ and $\mathrm{AA}$ can vary with different contexts such as, for example, when one is confronted with situations that are linked to alcohol use. However, with respect to the study procedures, the environment in which the participants were tested was not linked to the use of alcohol. Therefore, attentional and approach biases may be stronger in contexts that are more relevant to common drinking behavior. As such, perhaps we should not measure these biases in a treatment context. Instead, we should consider assessing these biases in a more naturalistic setting using, for example, ecological momentary assessment (EMA) methods.

Another argument for the use of EMA methods in future research is that monitoring behavior in daily life is more informative of the relapse process as a whole and can shed more light on the short-term predictive value of attentional and approach biases. A study using EMA methodologies has already shown that an AB toward drugs increases just before a relapse in a heroin-dependent inpatient sample (Marhe et al., 2013), indicating that AB may be a more proximal predictor of relapse, as suggested by Field and colleagues (2014). Future research is needed to investigate this further.

In summary, the present study did not find evidence for the long-term predictive value of $\mathrm{AB}$ and approach bias in alcohol-dependent outpatients. $\mathrm{AB}$ and $\mathrm{AA}$ for alcohol-related stimuli seem to be a feature of alcohol dependence, but they have little predictive value for relapse in the long term when measured during treatment. Future research should focus on the short-term predictive value of both $\mathrm{AB}$ and $\mathrm{AA}$ by adopting EMA methodologies to track these biases and examine their relationships to relapse in daily life.

\section{ACKNOWLEDGMENTS}

We thank Ivana van Berkel for her assistance with data collection. We also want to thank Professor Dr. Reinout Wiers for his advice on the reaction time tasks. Special thanks go to Brijder Verslavingszorg, their staff, and patients for their cooperation in our study.

\section{CONFLICT OF INTEREST}

The author(s) declare no potential conflict of interests with respect to the research, authorship, and/or publication of this article.

\section{REFERENCES}

American Psychiatric Association (2000) Diagnostic and Statistical Manual of Mental

Disorders. 4th ed. American Psychiatric Association, Washington, DC. 
Barkby H, Dickson JM, Roper L, Field M (2012) To approach or avoid alcohol? Automatic and self-reported motivational tendencies in alcohol dependence. Alcohol Clin Exp Res 36:361-368.

Christiansen P, Schoenmakers TM, Field M (2014) Less than meets the eye: reappraising the clinical relevance of attentional bias in addiction. Addict Behav 44:43-50.

Corbin WR, Zalewski S, Leeman RF, Toll BA, Fucito LM, O'Malley SS (2014) In with the old and out with the new? A comparison of the old and new binge drinking standards. Alcohol Clin Exp Res 38:2657-2663.

Cousijn J, Goudriaan AE,Wiers RW(2011) Reaching out towards cannabis: approach-bias in heavy cannabis users predicts changes in cannabis use.

Addiction 106:1667-1674.

CoxWM, Fadardi JS, Pothos EM (2006) The addiction-stroop test: theoretical considerations and procedural recommendations. Psychol Bull 132:443-476.

De Houwer J, Crombez G, Baeyens F, Hermans D (2001) On the generality of the affective Simon effect. Cogn Emot 15:189-206.

Eberl C, Wiers RW, Pawelczack S, Rinck M, Becker ES, Lindenmeyer J (2013) Approach bias modification in alcohol dependence: do clinical effects replicate and for whom does it work best? Dev Cogn Neurosci 4:38-51.

Eberl C, Wiers RW, Pawelczack S, Rinck M, Becker ES, Lindenmeyer J (2014)

Implementation of approach bias re-training in alcoholism-how many sessions are needed? Alcohol Clin Exp Res 38:587-594.

FieldM, Kiernan A, Eastwood B, Child R (2008) Rapid approach responses to alcohol cues in heavy drinkers. J Behav Ther Exp Psychiatry 39:209-218.

Field M, Marhe R, Franken IH (2014) The clinical relevance of attentional bias in substance use disorders. CNS Spectr 19:225-230.

Field M, Mogg K, Bradley BP (2005) Craving and cognitive biases for alcohol cues in social drinkers. Alcohol Alcohol 40:504-510.

Field M, Mogg K, Mann B, Bennett GA, Bradley BP (2013) Attentional biases in abstinent alcoholics and their association with craving. Psychol Addict Behav 27:71-80.

Franken IH (2003) Drug craving and addiction: integrating psychological and neuropsychopharmacological approaches. Prog Neuropsychopharmacol Biol Psychiatry 27:563-579.

Fuller RK (1997) Definition and diagnosis of relapse to drinking. Liver Transpl Surg 3:258262.

Garland EL, Boettiger CA, HowardMO (2011) Targeting cognitive-affective risk mechanisms in stress-precipitated alcohol dependence: an integrated, biopsychosocial model of automaticity, allostasis, and addiction. Med Hypotheses 76:745-754.

Garland EL, Franken IH, Howard MO (2012) Cue-elicited heart rate variability and attentional bias predict alcohol relapse following treatment.

Psychopharmacology 222:17-26.

Greenwald AG, Nosek BA, Banaji MR (2003) Understanding and using the Implicit

Association Test: I. An improved scoring algorithm. J Pers Soc Psychol 85:197-216.

Kersbergen I, Woud ML, Field M (2014) The validity of different measures of automatic alcohol action tendencies. Psychol Addict Behav 29:225-230.

Marhe R, Waters AJ, van de Wetering BJ, Franken IH (2013) Implicit and explicit drugrelated cognitions during detoxification treatment are associated with drug relapse: an ecological momentary assessment study. J Consult Clin Psychol 81:1-12.

Marlatt GA, Witkiewitz K (2010) Update on harm-reduction policy and intervention research. Ann Rev Clin Psychol 6:591-606.

Millisecond (2012) Inquisit.Millisecond Software, Seattle,WA.

Mogg K, Field M, Bradley BP (2005) Attentional and approach biases for smoking cues in smokers: an investigation of competing theoretical views of addiction. Psychopharmacology 180:333-341.

Moos RH,Moos BS (2006) Rates and predictors of relapse after natural and treated remission from alcohol use disorders. Addiction 101:212-222.

Ostafin BD, Palfai TP (2006) Compelled to consume: the Implicit Association Test and automatic alcohol motivation. Psychol Addict Behav 20:322-327. 
Palfai TP, Ostafin BD (2003) Alcohol-related motivational tendencies in hazardous drinkers: assessing implicit response tendencies using the modified- IAT. Behav Res Ther 41:1149_ 1162.

Roefs A, Huijding J, Smulders FT, MacLeod CM, de Jong PJ, Wiers RW, Jansen AT (2011) Implicit measures of association in psychopathology research. Psychol Bull 137:149-193.

Schoenmakers T, Wiers RW, FieldM(2008) Effects of a low dose of alcohol on cognitive biases and craving in heavy drinkers. Psychopharmacology 197:169-178.

Spruyt A, De Houwer J, Tibboel H, Verschuere B, Crombez G, Verbanck P, Hanak C, Brevers D, No€el X (2013) On the predictive validity of automatically activated approach/avoidance tendencies in abstaining alcohol-dependent patients. Drug AlcoholDepend 127:81-86.

Tiffany ST (1990) A cognitive model of drug urges and drug-use behavior: role of automatic and nonautomatic processes. Psychol Rev 97:1471168.

Waters AJ, Li Y (2008) Evaluating the utility of administering a reaction time task in an ecological momentary assessment study. Psychopharmacology 197:25-35.

Waters AJ, Marhe R, Franken IHA (2012) Attentional bias to drug cues is elevated before and during temptations to use heroin and cocaine. Psychopharmacology 219:909-921.

Wiers RW, Bartholow BD, van den Wildenberg E, Thush C, Engels RC, Sher KJ,Grenard J, Ames SL, Stacy AW(2007) Automatic and controlled processes and the development of addictive behaviors in adolescents: a review and a model. Pharmacol Biochem Behav 86:263-283.

Wiers RW, Eberl C, Rinck M, Becker ES, Lindenmeyer J (2011) Retraining automatic action tendencies changes alcoholic patients' approach bias for alcohol and improves treatment outcome. Psychol Sci 22:490-497.

Wiers RW, Rinck M, Dictus M, van den Wildenberg E (2009) Relatively strong automatic appetitive action-tendencies in male carriers of the OPRM1 G-allele.Genes Brain Behav 8:101-106.

World Health Organization (1997) Composite International Diagnosis Interview - Version 2.1.World Health Organization, Geneva. 
Snelleman, M., Schoenmakers, T.M., Mheen, D. van de. Attentional bias and approach/avoidance tendencies do not predict relapse or time to relapse in alcohol dependency. Alcoholism: Clinical and Experimental Research: 2015, 39(9), 1734

TABLES AND FIGURES

Table 1. Demographic Characteristics of Study Abstainers and Study Relapsers

\begin{tabular}{|c|c|c|c|c|c|c|}
\hline & \multicolumn{2}{|c|}{$\begin{array}{l}\text { Overall } \\
(n=50)\end{array}$} & \multicolumn{2}{|c|}{$\begin{array}{c}\text { Abstainers } \\
(n=30)\end{array}$} & \multicolumn{2}{|c|}{$\begin{array}{c}\text { Relapsers } \\
\quad(n=20)\end{array}$} \\
\hline & $M$ & SD & $M$ & SD & $M$ & SD \\
\hline Age & 48.7 & 10.7 & 50.3 & 11.5 & 48.5 & 9.8 \\
\hline Proportion men (in \%) & 77.8 & - & 75.0 & - & 66.7 & - \\
\hline \multicolumn{7}{|l|}{ Educational level (in \%) } \\
\hline High school & 24.0 & - & 20.0 & - & 30.0 & - \\
\hline Vocational education & 30.0 & - & 40.0 & - & 15.0 & - \\
\hline Higher vocational education & 22.0 & - & 16.7 & - & 30.0 & - \\
\hline University & 18.0 & - & 16.7 & - & 20.0 & - \\
\hline Other & 6.0 & - & 6.7 & - & 5.0 & - \\
\hline Daily alcohol consumption & 20.0 & 14.9 & 17.8 & 14.8 & 22.8 & 15.0 \\
\hline Number of previous treatments & 1.1 & 1.4 & 0.8 & 0.8 & 1.6 & 1.9 \\
\hline
\end{tabular}

Differences between groups on all variables were not significant (all ps $>0.14)$.

Table 2. D-Measure Mean Scores Per Group Per Task Per Session (R-SRC)

\begin{tabular}{|c|c|c|c|c|c|c|c|c|c|}
\hline & \multicolumn{3}{|c|}{ Overall } & \multicolumn{3}{|c|}{ Abstainers } & \multicolumn{3}{|c|}{ Relapsers } \\
\hline & $M$ & SD & $N$ & $M$ & SD & $N$ & $M$ & SD & $N$ \\
\hline AST session 1 & 0.314 & 0.353 & 48 & 0.339 & 0.313 & 28 & 0.278 & 0.408 & 20 \\
\hline AST session 2 & 0.140 & 0.370 & 42 & 0.12 & 0.42 & 29 & 0.20 & 0.24 & 13 \\
\hline R-SRC session 1 & -0.194 & 0.412 & 50 & -0.181 & 0.466 & 30 & -0.211 & 0.331 & 20 \\
\hline R-SRC session 2 & -0.02 & 0.35 & 43 & 0.02 & 0.37 & 30 & -0.12 & 0.27 & 13 \\
\hline
\end{tabular}

AST, Addiction Stroop Task; R-SRC, relevant Stimulus-Response Compatibility Task. 